\title{
Forkhead box P3 gene expression and chromosomal analysis in a sample of Iraqi patients with multiple sclerosis
}

\author{
Zainab A. Aljawadi* \\ Akram M. Almahdawi** \\ Mahmood A.N. Kashmoola*** \\ Ban A. Abdul-Majeed****
}

\author{
MBChB, PhD \\ FIBMS \\ FIBMS \\ $\mathrm{MBChB}, \mathrm{PhD}$
}

Abstract:

Background: Multiple Sclerosis disease is a demyelination process which interferes with the neuronal signal transmission, thus leading to different cognitive and physical dysfunctions like optic neuritis, motor, sensory and coordination problems. Recently many researches have been directed toward studying the relation between some genes and multiple sclerosis. Among the important genes to be studied in multiple sclerosis is the forkhead box P3 gene expression.

Fac Med Baghdad 2017; Vol.59, No.1

Received: Oct. 2016

Accepted: Nov.2016
Objectives: The aims of the present work were to study the expression of forkhead box P3 gene by real time polymerase chain reaction, and to perform chromosomal analysis on the multiple sclerosis patients peripheral blood lymphocytes.

Patients and methods: A case-control study was performed using real time polymerase chain reaction technique to measure the relative expression of forkhead box P3 gene in peripheral blood leukocytes of 25 newly diagnosed untreated multiple sclerosis patients and comparing them with that of 25 apparently healthy controls, in addition to performing chromosomal analysis on multiple sclerosis patients peripheral blood lymphocytes.

Results: Significant down-regulation in forkhead box P3 gene was detected in multiple sclerosis patients in comparison to controls. Chromosomal analysis that was performed for multiple sclerosis patients revealed normal results.

Conclusion: Multiple sclerosis is associated with significant change in forkhead box P3 gene expression that can be measured by real time polymerase chain reaction technique. Furthermore, multiple sclerosis is not associated with gross chromosomal abnormalities.

Key words: Multiple sclerosis, Foxp3 gene, real time PCR, chromosomal abnormality.
Introduction:

Multiple Sclerosis (MS) disease is a demyelination process which interferes with the neuronal signal transmission, thus leading to different cognitive and physical dysfunctions like optic neuritis, motor, sensory and coordination problems $(1,2,3)$. The exact cause of MSis unknown, but epidemiologic studies hypothesized that a complex interaction is present between environmental and genetic factors (4). Recently many researches have been directed toward studying the relation between some genes and MS. Among the important genes to be studied in MS is Foxp3 gene expression, where in one study theMesenchymal stem cell (MSC) therapy was applied on a group of MS patients. After Six months following intrathecal injection of MSC, a significant increase in Foxp3 expression was detectedwhich was also accompanied by clinical stability of the

* Dept. of Microbiology, Medical college, Baghdad University. Email: Microbiologist1982@yahoo.com

** Dept. of neurology in Baghdad Teaching Hospital .

*** Dept. of neurology, Al-Mustansiriyah Medical College.

**** Department of Pathology in Medical college of Al-Nahrain University. patients (5). Furthermore, transduction of myelin proteolipid protein specific autoreactive-T-cells with Foxp3 inhibits their ability to trigger experimental autoimmune encephalomyelitis. This supports the fact that Foxp3 inhibits autoreactive-T-cells effector function (6). Foxp3 protein is used in immune system responses where it has a crucial role in controlling the function and development of natural CD4 +/CD25 + Treg cells which are involved in active suppression of inappropriate immune responses $(7,8)$. The Foxp3 exerts its effect by occupying the promoters of genes that control the function of Tregcell, It may also inhibit the transcription of crucial genes following $\mathrm{T}$ cell receptors stimulation (9). Regarding chromosomal abnormalities, it is well known that abnormalities which affect the chromosome either entirely or affect large region of it that covers multiple genes are capable of affecting human health. Therefore, chromosomal analysis is important to be checked in MS patients. Anyhow, it is unlikely for MS disease to be caused by an inherent chromosomal abnormality, on contrast, some studies showed that somatic chromosomal aberrations were found in the cells of MS patients in higher frequencies 
than in controls $(10,11)$. One study reported that chromosomal abnormality are more likely to be found in MS patients who had more frequent relapses or progressive type of MS (12). Another study reported that CSF lymphocytes showed more chromosomal aberration than peripheral blood lymphocytes in MS patients (13).

However, most of the abnormalities reported by these studies were an increase in the incidence of breaks, rearrangements, and gaps in the chromosomes of MS patients in comparison to controls (11). Such abnormality is not necessary to be found in MS patients, where one study did not found any difference in the occurrence of chromosomal breaks in blood lymphocytes between MS patients and controls (14).

\section{Method}

$\backslash$ A case control study was performed using two steps reverse transcription polymerase chain reaction technique to study the Foxp3 gene expression in patients with MS disease as well as performing a chromosomal analysis on their peripheral lymphocytes. The study included twenty five patients who were newly diagnosed as having MS disease and before receiving any steroid treatment or immunomodulatory therapy who attend Multiple Sclerosis Clinic, or are admitted to the Neuromedical ward in Baghdad Teaching Hospital, and Special Nursing Home in Baghdad Medical City as well as from the Neuromedical Consultancy Unit in Al-Yarmouk Teaching Hospital in Baghdad city. Multiple sclerosis diagnosis was achieved according to the 2010 McDonald criteria (15) by the neurologist senior depending on clinical history, physical examination, brain and spinal cord MRI, Visual evoked potential if required, and laboratory tests as necessary to exclude other demyelinating diseases and other possible MS differential diagnosis. The patients were asked for their permission to give blood samples and signed an informed consent for their agreement to participate in the research project. Data information for each patient were collected including clinical history, Physical signs, Laboratory tests, and MRI findings. Regarding controls, they included twenty five apparently healthy medical staff with no history of having any autoimmune disease or receiving any steroid or immunomodulatory therapy.

All the studied blood samples of the patients and controls were collected, tested, and analyzed during the period from $1^{\text {st }}$ of April 2015 to $31^{\text {st }}$ of March 2016. The samples included $5 \mathrm{mls}$ of blood which were collected from the participant under aseptic technique, $2 \mathrm{mls}$ of them were evacuated into K3EDTA blood tube (Human, Germany) to be followed with their suitable processing for measuring Foxp3 genes expression. The remaining $3 \mathrm{mls}$ were poured into a heparinized blood tube for chromosomal analysis. All samples were collected in time period between $9 \mathrm{am}-12 \mathrm{am}$ for both the MS patients and healthy controls in order to avoid measuring the effects of diurnal variation on immune function.

A molecular experiment was conducted to find foxp3 gene expression of the peripheral white blood cell for both the patients and controls. This gene expression in each individual was normalized to the expression of one of his/ her reference genes which was RNU6-2 gene that was also obtained from their peripheral white blood cell. After finding the foxp3 gene expression for each participant and normalizing it with his/ her own reference genes, they were all compared to the normalized target gene expression of a single calibrator (that was considered to be equal to 1 ) in order to find the final fold change in foxp3 gene expression. In details, this experiment included the following steps:

Leukocytes separation by the use oferythrocyte lysis Buffer (Qiagen, Germany) according to the manufacturer protocol (16).

Purification of Total RNA which was performed by the use of miRNeasy Mini Kit (Qiagen, Germany). This Kit combines phenol/guanidine based lysis of samples and silicamembranebased purification of total RNA. These steps were performed according to the manufacturer's protocol (16).

Assessment of the extracted RNA purity and concentration that were automatically measured by micro-volume UV spectrophotometer. The purity was measured for each sample depending on the absorbance at $260 \mathrm{~nm}\left(\mathrm{~A}_{260}\right)$ and $280 \mathrm{~nm}$ $\left(\mathrm{A}_{280}\right)$ ratio $\left(\mathrm{A}_{260} / \mathrm{A}_{280}\right)$. The sample which had RNA purity of 1.9 - 2.1 was accepted and included in the study (17).

Two-step- RT-PCR reaction: Total RNA was the starting material in RT-PCR reaction which was performed in two steps.

Step 1- RNA Reverse Transcription: Measuring the expression of target genes by qPCR requires the conversion of RNA intocDNA through the process of reverse transcription that was done by the use of miScript II RT Kit (Qiagen, Germany). All the steps were conducted according to manufacturer instructions (17).

Step 2- Real-time PCR: Following the conversion of RNA into cDNA, real time PCR reaction was conducted on the cDNA template by the usage of SYBR Green reporter to detect the amplified target. For detection of mRNA, cDNA prepared in a reverse transcription reaction serves as a template for real-time PCR analysis using a QuantiTect Primer Assay in combination with QuantiTect SYBR Green PCR Master Mix which is provided in the miScript SYBR Green PCR Kit (Qiagen, Germany). The reaction setup was prepared according to the manufacturer's recommendations (17). At the end of qPCR reaction, analysis of data was performed. Mx3000P software associated with the Stratagene Agilent real time PCR machine was used to find $\mathrm{C}_{\mathrm{T}}$ values and to demonstrate the amplification plots as well as the dissociation curves for each reaction. Once 
data capture completed, expression analysis was performed by the relative quantification.

\section{Statistical analysis:}

The results were analyzed by the use of Statistical-Package for the Social Sciences (version 23.0 software), and the Microsoft-Office Excel software, 2007. Independent T- test, Mann-Whitney U test, were used to calculate the significance of the differences. $\mathrm{P}$ value less than 0.05 was considered to be a statistically significant.

Chromosomal analysis: Chromosomal study was done in the Cytogenetic Department of Teaching Laboratories in Baghdad Medical City (lymphocytes cultivation, and slides preparation, and staining) and in Al Nahrain Faculty of Medicine (Microscopical examination of slides).

The peripheral blood lymphocytes karyotyping method was performed to provide information about the possibility of chromosomal abnormalities in MS patients. Chromosome medium P (complete medium) (Euro Clone, Italy) was used for the cultivation of human peripheral blood lymphocytes. The lymphocytes do not undergo subsequent cell divisions under normal condition. Therefore, they were stimulated by phytohemagglutinin (PHA) to start mitosis and DNA replication. The colcemidsolution whichacts as a mitotic inhibitor was added to the culture media to stop mitosis at the metaphase stage.Trypsin solution and Giemsa stain were used in the staining procedure of the chromosomes $(18,19,20)$. The slides were examined using the magnification Power
$1000(100 \times 10)$ by the oil immersion lens. Generally, 20 cells were analyzed to rule out mosaicism. The chromosomes were counted, examined carefully for possibility of presence of any gross abnormality like breaks, circular chromosomes, or dicentric chromosomes. The results were recorded.

\section{Results:}

From a total of 25 patients with MS, $23(92 \%)$ were females and $2(8 \%)$ were males with a female to male ratio (F:M) of 11.5:1. The ages of these patients ranged between $18-55$ years. the mean age was 33.32 years with a standard deviation of \pm 9.45 years. The controls group was selected to be sex and age matched with the patients group, where they also consisted of $23(92 \%)$ females and $2(8 \%)$ males with a female to male ratio (F:M) of 11.5:1. The mean age of the controls group was 33.84 years with a standard deviation of \pm 9.05 years, and range of 19-54 years. Regarding the type of MS, there was only two type of patients. Relapsing remitting MS patients comprised $23(92 \%)$ individual, and the remaining $2(8 \%)$ patients were of primary progressive MS type.

The mean rank of the Foxp3 gene Log fold change values for patients (21.48) was lower than that for controls (29.52). The observed difference was statistically significant where the p-value was less than 0.05 . This indicates that Foxp3 gene expressions in the patients were significantly down-regulated in comparison to the control (figure 1). Effect size for Foxp3 gene expression relation between patients and controls was also tested, where $\mathrm{r}$ was $(-0.275)$.
2 Log fold change values of Foxp3 gene RNA of the Patients

2 Log fold change values of Foxp3 gene RNA of the controls

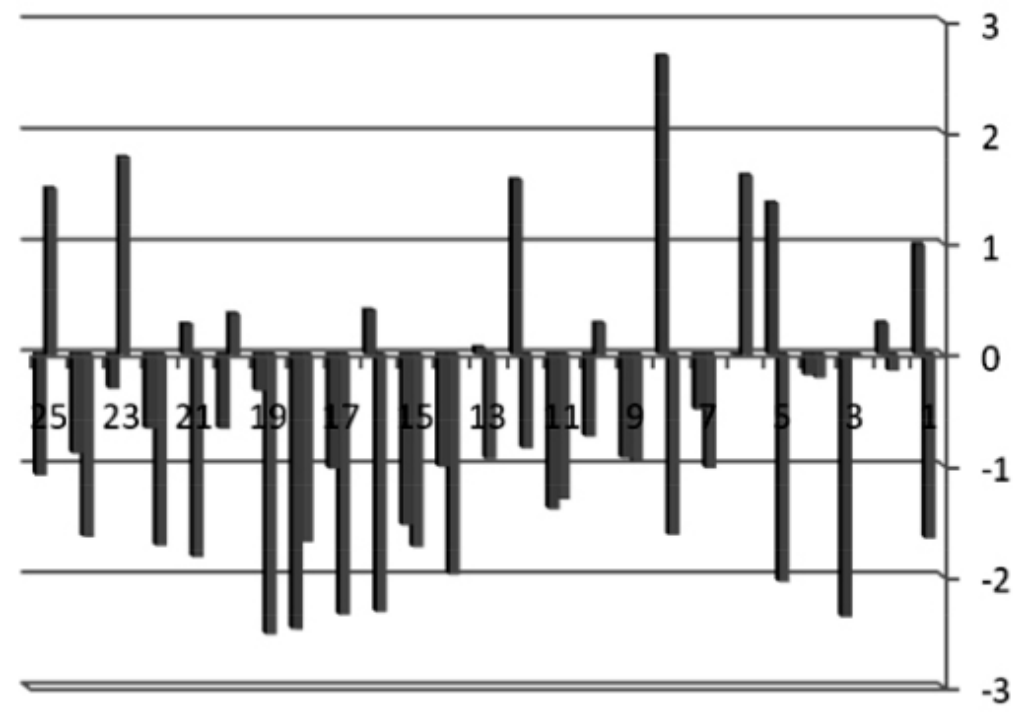

Figure 1: Foxp3 gene expressions in patients and controls 
This figure shows Foxp3 gene expressions in patients and controls, where the Bars which are above the zero line refer to the genes up-regulation, while those below it represent genes down-regulation. The blue bars are for patients, while the red bars are for controls.For the mean rank of foxp3 gene Log fold change values in the female patients, it was lower than that of the male patients but it failed to reach the statistical significance, where the P-value was more than 0.05 .

Foxp3 gene expression inMS patients with presentation periods of less than one month had mean of Log fold change values lower than those having presentation period of one month and more. The P-value was less than 0.05 indicating the presence of a statistically significant difference in Foxp3 gene expression which was down-regulated in patients who presented in period less than one month in comparison with those of one month and more. Effect size for the means difference in Foxp3 gene expression between these two groups was also tested, where Cohen's d was (-0.786).

For the attacks interval (between the first and second attack), Foxp3 gene expression showed lower mean of the Log fold change values in MS patients with attacks interval less than one year $(-1.38)$ in comparison to those having attack interval of one year and more $(-0.56)$. The P-value was less than 0.05 indicating the presence of a statistically significant difference in Foxp3 gene expression which was down-regulated in patients with attacks interval less than one year in comparison with those of one year and more. Effect size for the means difference in Foxp3 gene expression between these two groups was also tested, where Cohen's d was (-0.698).

There was no statistically significant differences in the means rank of the Log fold change values of Foxp3 genes expression between the relapsing remitting MS (RRMS) and primary progressive MS (PPMS) where the P-value was more than 0.05 .

Chromosomal Analysis: All the studied MS patients (except one patient who had failure of growth of her lymphocytes culture) had normal karyotyping, XY 46 chromosomes for males (figure 2 ), and XX 46 chromosomes for females (figure 3), no breaks, circular, or dicentric chromosomes were detected.

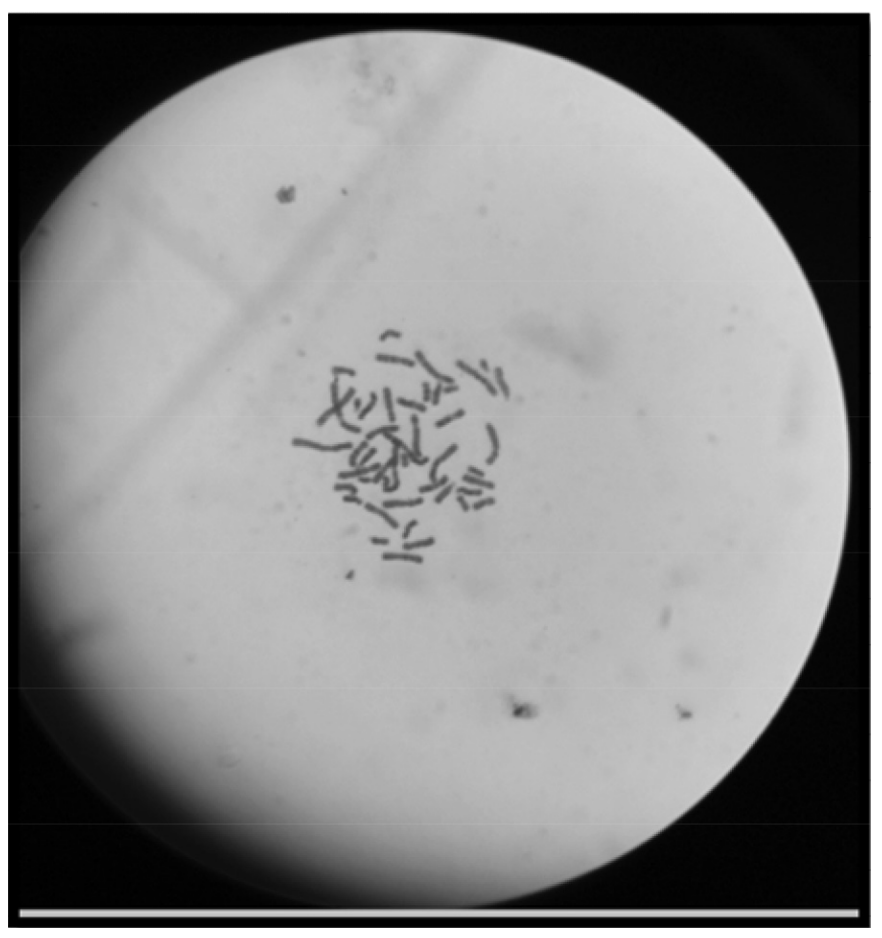

Figure 2: Male karyotyping with XY, 46 Chromosomes At magnification power of $10 \times 100$

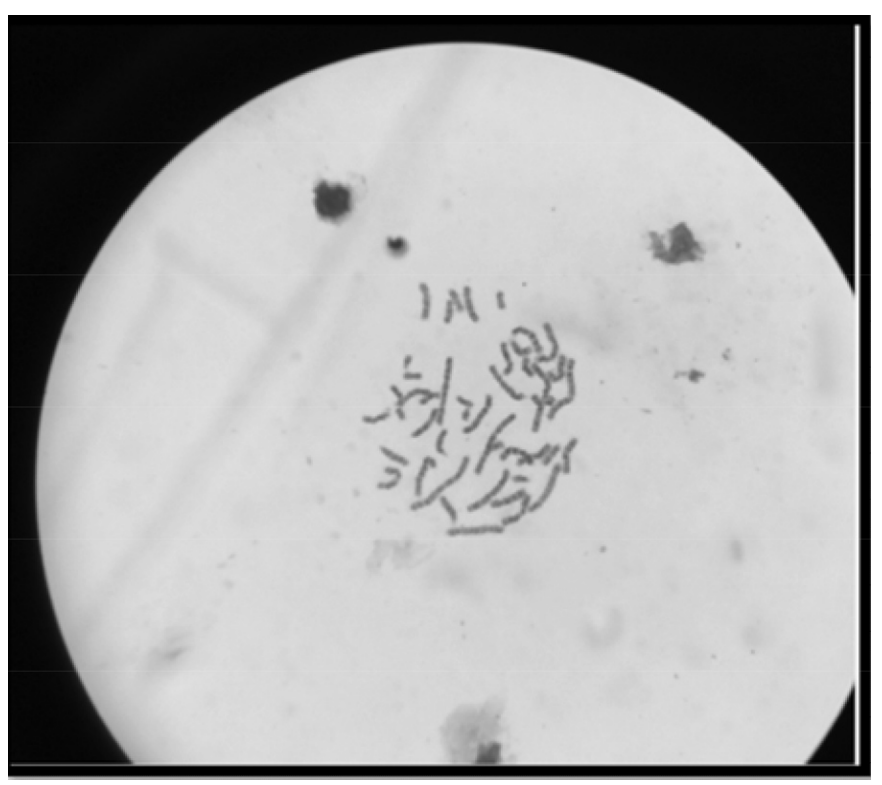

Figure 3: Female karyotyping with $\mathrm{XX}, 46$ Chromosomes At magnification power of $10 \times 100$ 


\section{Discussion}

Multiple sclerosis is a disease characterized by inflammatory demyelination of the CNS (21)Although the features of the pathological lesions were described since 150 years ago (22), the exact etiology and the pathophysiology still need to be elucidated. However, advances in the genetic, immunological, and pathological techniques play a role in increasing our understanding for the mechanism of the disease. The female to male ratio (F:M) was $11.5: 1$. This higher percent of MS in female in comparison to male was consistent with the reports of other studies $(23,24)$ who found also a higher prevalence of the disease in females. But, female to male ratio (F:M) which is 11.5:1 was somewhat higher than the reported ratio by other studies which reported range of F:M ratios of (2.3$3.5: 1)(23,24,25)$. Such differences in the ratio could be due to the selected criteria for the included patients in this study, where only the newly diagnosed cases with no previous steroid treatment were included, thus it does not reflect the exact F:M ratio for the disease. However, Many factors may play a role in the difference in sex prevalence including the genetic differences, the effect of gonadal hormones, differences in modern lifestyle and environmental exposures in men and women (26,27). For Foxp3 gene, there was a statistically significant difference in its expression which was lower in MS patients in comparison to controls. This was in accordance with another study which also documented down regulation of Foxp3 gene in MS patients (28). This gene down-regulation could be attributed to the hyper-methylation of Foxp3 gene in MS patients as reported by one study (29). The expansion of pathogenic T-cell in MS disease may represent the sequel of impairment of immune regulatory mechanism that is achieved by Tregcells $(28,30)$. Since Foxp3 is considered a specific transcription factor for Tregcells(31), thus the down-regulation of Foxp3 gene in MS patients support the association of Treg impairment with MS pathogenesis. However, this significant down-regulation of Foxp3 gene in MS patients in comparison to controls with small effect size $(r=-0.28)$ make it a biological marker in MS diagnosis with diagnostic accuracy AUC of (0.66), sensitivity (68\%), and specificity (64\%) at cut off point $(\leq-0.79)$ fold change.In this work, there were no statistically significant differencesin Foxp3 gene expression in MS patients according to sex. Similar studies with such classification to be compared with, were not found. This indicates that this gene is unlikely to be responsible for the sex bias in the MS disease. Foxp3 genes hadstatistically significant differences in their expression between MS patients who presented in period less than one month, and those in period of one month and more. Similar studies were not available. The timing of MS clinical presentations did notreflect the state of underlying inflammation at once because it related to the process of demyelination $(1,2,3)$. Moreover, despite some inflammatory processes do occur but they are not necessary to be associated instantly with a clinical feature (3). On the other hand, the time at which the patients undergo clinical improvement is not reflecting the time of the inflammatory reaction stoppage, but it is mainly due to the axonal redistribution of the $\mathrm{Na}$ channels, and possibly later on due to re-myelination process $(2,3)$. Therefore, it is necessary to find a biological markersthat can give an idea about the state of inflammation. Foxp3 gene was significantly down-regulated in patients with shorter presentation time in comparison to those with presentation period of one month and more. This finding possibly make this gene a suitable biological marker to follow up the state of immunological process in MS patients.On the other hand, Foxp3 gene had a statistically significant difference in its expression which was down-regulated in patients with attacks interval less than one year in comparison to those of one year and more. Similar study with such finding is not available. This indicatesthat patients with lower expression of Foxp3 gene are susceptible to more MS attacks than those with higher expression. This significant difference in Foxp3 gene expression with a medium effect size ( cohen's $d=-0.698$ ) make it possibly a good biological marker for expectation of MS patients prognosis regarding attacks interval. The diagnostic accuracy for using Foxp3 gene as a biological marker for predicting MS attacks interval (less than one year or one year and more) was significant with AUC of ( 0.66$)$, sensitivity of $(81 \%)$, and specificity of $(65 \%)$ at cut off point of (-0.99) fold change. This study included two types of MS, the RRMS and PPMS. Foxp3 gene did not show any significant difference in its expressions between these two types. Another study with such finding was not available. This finding indicates that this gene is probably not associated with the bias toward one of these two types, RRMS or PPMS. Regarding chromosomal analysis, the chromosomal study of the MS patient reveals normal chromosomal count. Chromosomal breaks, circular, or dicentric chromosomes were not detected. This was in consistent with another research which reported a similar finding (14). Such finding indicates that the underlying MS pathology is probably not due to gross chromosomal defect that can be detected by a light microscope. On the contrary, another study reported an increase in the incidence of chromosomal breaks in MS patients $(2.0 \%)$ in comparison to control $(1.1 \%)(11)$. However, such difference in findings could be due to difference in type of MS, difference in relapse rate, or exposure of patients to chemical or drugs that may affect the chromosomal structure (10).

\section{Authors Contributions:}

Zainab A. Aljawadi: Study conception, design, samples collections and processing, data analysis and interpretation, and writing the research.

Akram M. Almahdawi :Consultant neurologist provided the 
clinical cases of multiple sclerosis patients from Baghdad medical city

Mahmood A. Kashmoola: Specialist neurologist provided the clinical cases of multiple sclerosis patients from Al-Yarmouk Teaching Hospital

Ban A. Abdul-Majeed : Supervisor, critical revision

\section{References}

1-Leary S, Giovannoni G, Howard R, Miller D, Thompson A. Multiple Sclerosis and Demyelinating Diseases. In: Clarke C, Howard R, Rossor M, Shorvon S (eds.) .Neurology A Queen Square Textbook(1st ed.). London: Blackwell Publishing Ltd,2009,pp. 411-447.

2-Hauser SL ,Goodin DS. Multiple sclerosis and other demyelinating diseases. In: Hauser SL (ed.). Harrisons neurology in clinical medicine. 2nd ed. London: McGraw-Hill companies, 2010, pp.435-450.

3- StankiewiczJ .Multiple sclerosis and other inflamatory demyelinating diseases. In: Ropper AH, Samuels MA, Klein JP (eds.). Adams and Victor's PRINCIPLES OF NEUROLOGY (10th ed.). London: McGraw-Hill education, 2014,pp. 915945.

4- Ascherio A, Munger KL. Environmental risk factors for multiple sclerosis. Part I: the role of infection. Annals of neurology. 2007 Apr 1;61(4):288-299.

5-Mohajeri M, Farazmand A, Bonab MM, Nikbin B, Minagar A. FOXP3 gene expression in multiple sclerosis patients preand post mesenchymal stem cell therapy. Iranian Journal of Allergy, Asthma and Immunology. 2011 Sep 1;10(3):155-161. 6- Bettelli E, Dastrange M, Oukka M. Foxp3 interacts with nuclear factor of activated $T$ cells and $N F-\kappa B$ to repress cytokine gene expression and effector functions of $T$ helper cells. Proceedings of the National Academy of Sciences of the United States of America. 2005 Apr 5;102(14):5138-5143.

7- Zhang L, Zhao Y. The regulation of Foxp3 expression in regulatory $C D 4+C D 25+T$ cells: multiple pathways on the road. Journal of cellular physiology. 2007 Jun 1;211(3):590597.

8- Ricciardelli I, Lindley KJ, Londei M, Quaratino S. Anti tumour necrosis $\square \alpha$ therapy increases the number of FOXP3+ regulatory $T$ cells in children affected by Crohn's disease. Immunology. 2008 Oct 1;125(2):178-183.

9- Marson A, Kretschmer K, Frampton GM, Jacobsen ES, Polansky JK, MacIsaac KD, Levine SS, Fraenkel E, von Boehmer H, Young RA. Foxp3 occupancy and regulation of key target genes during T-cell stimulation.Nature. 2007 Feb 22;445(7130):931-935.

10- Unglaub-Leisten I, Stickl H, Frick E, Angstwurm H, Ring $J$. [Chromosomal aberrations in peripheral lymphocytes of patients with multiple sclerosis (author's transl)]. Deutsche medizinischeWochenschrif). 1975 Oct;100(40):2028-2034.
11- Vormittag W, Maida E, Totseva A, Kunze-Mühl E, Summer $K$. [Multiple sclerosis and chromosomal aberrations (author's transl)]. WienerklinischeWochenschrift. 1981 Dec;93(23):724729.

12- D'Alessandro E, Cola MD, Lo Re ML, Ligas C, Vaccarella $C$, D'Andrea F, Marini C, Prencipe M. Nonrandom chromosome changes in multiple sclerosis. American journal of medical genetics. 1990 Nov 1;37(3):406-411.

13- Sandberg-Wollheim M, Kristoffersson U, Mandahl N, Högstedt B. Increased frequency of chromosome aberrations in long-term cultured cerebrospinal fluid lymphocytes of patients with multiple sclerosis. Journal of the neurological sciences. 1991 Mar 31;102(1):46-50.

14- Petcu I, Savu D, Vral A, Thierens H, Nagels G, De Ridder L. Chromosomal Radiosensitivity in Secondary $\square$ Progressive Multiple Sclerosis Patients. International journal of radiation biology. 2004 Sep 1;80(9):663-672.

15-Polman CH, Reingold SC, Banwell B, Clanet M, Cohen JA, Filippi M, Fujihara K, Havrdova E, Hutchinson M, Kappos L, Lublin FD. Diagnostic criteria for multiple sclerosis: 2010 revisions to the McDonald criteria. Annals of neurology. 2011 Feb 1;69(2):292-302.

16- miRNeasy Mini Kit handbook [package insert]. Germany: Qiagen; 2014

17- miScript PCR System Handbook [package insert]. Germany: Qiagen; 2011

18- Watt JL, Stephen GS. Lymphocyte culture for chromosome analysis. In: Rooney ED, Czepulkowski BH (eds.). Human Cytogenetics: a practical approach. Oxford: IRL Press, 1986, pp.39-55.

19- Block AW. Cancer cytogenetics. In: Gersen SL, Keagle $M B$ ( eds).The principles of clinical cytogenetics. Totowa: Humana Press, 1999, pp. 345-420 .

20- Howe B, Umrigar A, Tsien F. Chromosome preparation from cultured cells. Journal of visualized experiments: JoVE. 2014; 28(83): Available from doi: 10.3791/50203.

21 - Brück $W$. The pathology of multiple sclerosis is the result of focal inflammatory demyelination with axonal damage. Journal of neurology. 2005 Oct 1;252(5):v3-9.

22- Orrell RW. Multiple Sclerosis: The History of a Disease. Journal of the Royal Society of Medicine. 2005 Jun 1;98(6):289.

23-Compston A, Coles A. Multiple sclerosis. Lancet. $2002 \mathrm{Apr}$ 6;359(9313):1221-1231.

24-Ahlgren C, Odén A, Lycke J. High nationwide prevalence of multiple sclerosis in Sweden. Multiple Sclerosis Journal. 2011 Aug 1;17(8):901-908.

25- Wallin MT, Culpepper WJ, Coffman P, Pulaski S, Maloni H, Mahan CM, Haselkorn JK, Kurtzke JF, Veterans Affairs Multiple Sclerosis Centres of Excellence Epidemiology Group. 
The Gulf War era multiple sclerosis cohort: age and incidence rates by race, sex and service. Brain. 2012 Jun 1;135(6):17781785 .

26- Greer JM, McCombe PA. Role of gender in multiple sclerosis: clinical effects and potential molecular mechanisms. Journal of neuroimmunology. 2011 May 31;234(1):7-18.

27- Harbo HF, Gold R, Tintoré M. Sex and gender issues in multiple sclerosis. Therapeutic advances in neurological disorders. 2013 Jul 1;6(4):237-248.

28-Huan J, Culbertson N, Spencer L, Bartholomew R, Burrows GG, Chou YK, Bourdette D, Ziegler SF, Offner H, Vandenbark AA. Decreased FOXP3 levels in multiple sclerosis patients. Journal of neuroscience research. 2005 Jul 1;81(1):45-52.

29- Li X, Xiao B, Chen XS. DNA Methylation: a New Player in Multiple Sclerosis. Molecular Neurobiology.2016 Jun 17:111 .

30- Zozulya AL, Wiendl $H$. The role of regulatory $T$ cells in multiple sclerosis. Nature clinical practice Neurology. 2008 Jul 1;4(7):384-398.

31-Rudensky AY. Regulatory T cells and Foxp3. Immunological reviews. 2011 May 1;241(1):260-268. 